\title{
Ahordagens de Cooperação e Colahoração na Utilização de Ambiente de Aprendizagem Mediado por Computador pelos Portadores de Necessidades Educacionais Especiais com Limitação Visual
}

Boardings of Cooperation and Contribution in the Use of Environment of Learning Mediated by Computer for the Special Carriers of Educational Necessities with Visual Limitation 
Resumo: Este artigo trata da utilizaÁ,o de Ambiente de Aprendizagem Mediado por Computador (AAMC), enfocando os Portadores de Necessidades Educacionais Especiais (PNEEs) no processo de ensino e de aprendizagem. O uso de ferramentas computacionais que atendam ao desenvolvimento da comunicaÁ,,o, ‡ interaÁ,,o, $\ddagger$ colaborá́,,o e $\ddagger$ cooperaÁ,,o para a superaÁ,,O de suas limitaÁles e a construÁ,,o de conhecimento torna significativo o processo de inclus,, o social em uma sociedade de excluldos.

Palavras-chave: EducaÁ,o especial. Inform·tica na educaÁ,,o. Ambiente de aprendizagem mediado por computador. CooperaÁ,,o. ColaboraÁ,,o.

Abastract: This is an article about the use of Environment of Learning Mediated by Computer, focusing the Bearers of Special Education Needs and their teaching and learning process. The use of computing tools that permit the development of communication, interaction, collaboration and cooperation for overcoming their limitations and knowledge construction turns significant the process of social inclusion into a society of excluded.

Keywords: Special education. Computer science in the education. Environment of learning mediated by computer. Cooperation. Collaboration.

ESTABEL, Lizandra Brasil; MORO, Eliane L. da Silva; SANTAROSA, Lucila Maria Costi. Abordagens de CooperaÁ,o e ColaboraÁ,o na UtilizaÁ,,o de Ambiente de Aprendizagem Mediado por Computador pelos Portadores de Necessidades Educacionais Especiais com LimitaÁ,,o Visual. Informática na Educação: teoria \& prática, Porto Alegre, v. 6, n. 1, p. 41-54, jan./jun. 2003. 


\section{Introdução}

Nos dias atuais, surge, dentre outras, uma nova concepÁ,,o sobre o papel que a escola representa na sociedade: deve ser um espaÁo inclusivo, que atenda $\ddagger$ s diversidades e que propicie uma educaÁ,,o de qualidade, apresentando respostas $\ddagger s$ necessidades de seus educandos. Para atingir esses objetivos, È necess-rio que esteja preparada para atender as necessidades educacionais de seus alunos, inclusive os Portadores de Necessidades Educacionais Especiais (PNEEs).

Os Portadores de Necessidades Educacionais Especiais, segundo a DeclaraÁ,,O Mundial sobre EducaÁ,,o para Todos (2003) ìs, o considerados tanto como cidad, ,os comuns quanto como cidad,os peculiares: cidad, ,os comuns ao se propor que o acesso ‡ educaÁ,,o como eq, idade seja universalizado para todos (Art. $3 \infty$ ) e peculiares ao explicitar-se que È preciso garantir-lhes igualdade de acesso $¥$ informaÁ,,o como parte integrante do sistema educativo, independente do tipo de deficiĺncia que possuam (Art. $5 \infty$ )î. Portanto, faz-se necess-rio que educadores e aprendizes sejam incentivados a trabalharem juntos, cooperativamente, na superaÁ,,o das dificuldades.

O Ambiente de Aprendizagem Mediado por Computador (AAMC) deve possibilitar que ambos trabalhem conjuntamente, utilizando ferramentas de comunicaÁ,,o adaptadas $\ddagger s$ suas necessidades. No caso dos alunos PNEEs com limitaÁ,o visual, as ferramentas e o ambiente devem apresentar adaptaÁıes para que a falta de vis,,o deixe de ser uma deficiĺncia, que pode ser vista como incapacidade, e passe a ser uma limitaÁ,„o, que pode ser superada com a utilizaÁ,,o das ferramentas adequadas e com a ajuda dos pares, que s, o os integrantes do grupo, educadores e aprendizes.

Portanto, este artigo trata da possibilidade de utilizaÁ,o de um Ambiente de Aprendizagem Mediado por Computador pelos portadores de limitaÁ,o visual e os seus educadores, em um processo de comunicaÁ,,o, interaÁ,,o, colaboraÁ,,o e cooperaÁ,,o para a superaÁ,,o de suas limitaÁıes e construÁ,o de conhecimento.

\section{0 acesso à informação pelos PNEEs com limitação visual}

O surgimento da escrita data de trís a cinco mil anos antes da era Crist,.. No entanto, somente no sÈculo XIX os portadores de limitaÁ,,o visual passaram a ter acesso a escrita, o que permitiu que fossem incluldos na chamada cultura letrada. Em 1825, criado por Louis Braille, surge um sistema de leitura t·til e escrita para portadores de limitaÁ,,o visual que recebe o seu nome - Sistema Braille.

... nossa responsabilidade fazer do sistema Braille n„o um sistema fechado e antisocial, mas mostr--lo ao mundo como uma marca de cultura, um modo de vis, o de mundo, a senha matriz de nossa emancipaÁ, o social e da luta pela nossa cidadania. (BELARMINO, 2001).

O sistema Braille È formado por seis pontos em relevo, que apresentam 63 combinaÁıes. Com ele È possìvel fazer letras, $\mathrm{n}$ ' meros, sìmbolos quìmicos e matem ticos

[...] pensar numa coisa fascinante. Em um, talvez dois segundos, nesse gesto de premir seis pontos em um papel em branco, Braille nos conectou com a nossa cultura escrita. Com um clique de nada, Braille criou as chaves para a nossa inclus,,o em v rios outros mundos: os profissionais, os educacionais, os tecnolÛgicos, outros mundos estèticos, afetivos e prazerosos atĖ ent,,o desconhecidos de nÛs. (BELARMINO, 2003). 


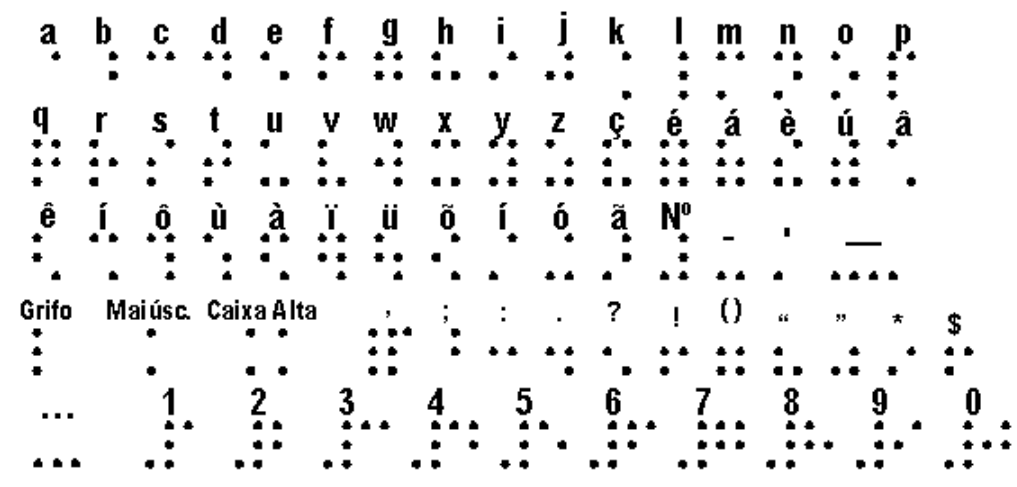

Figura 1 - Alfabeto Braille

Fonte: http://intervox.nce.ufrj.br/ fabiano/Braille.htm

Para o registro das informaÁıes È necess·ria a utilizaÁ,o da reglete, duas placas de metal ou pl-stico, fixas em um dos lados por dobradiÁas, de modo a permitir a introduÁ,,o do papel. Com o punÁ,,o, o portador de limitaÁ,o visual faz pon- to por ponto atÈ formar o sìmbolo. $\mathrm{Na}$ reglete, escreve-se da direita para a esquerda, no entanto, ao virar o papel, a leitura Ė feita normalmente, da esquerda para direita. Essa forma de registro È feita individualmente.

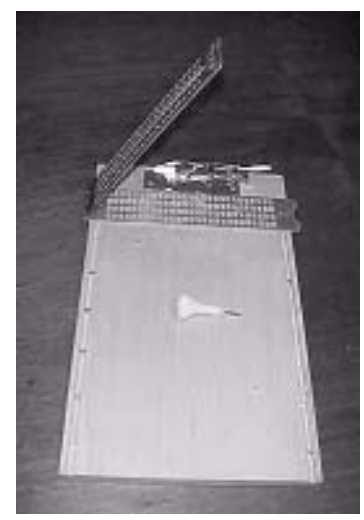

Figura 2 - Reglete e punÁ,,O

Fonte:http://intervox.nce.ufrj.br/ fabiano/Braille.htm

Existem tambÈm $\mathrm{m} \cdot$ quinas especiais de datilografia que registram em Braille. No entanto, com o avanÁo da inform·tica, surgiram as impressoras em Braille, que possibilitaram a reproduÁ,,o de obras com maior rapidez, gerando um processo de democratizaÁ,o da informaÁ,o.
Apesar desse avanÁo, faz-se neces$s \cdot$ rio um investimento financeiro em equipamentos e softwares, muitas vezes invi-vel para as pessoas portadoras de necessidades especiais devido ao elevado custo. Para o PNEEs com limitaÁ,o visual o acesso ‡ informaÁ,,o ainda apresenta muitos impe- 


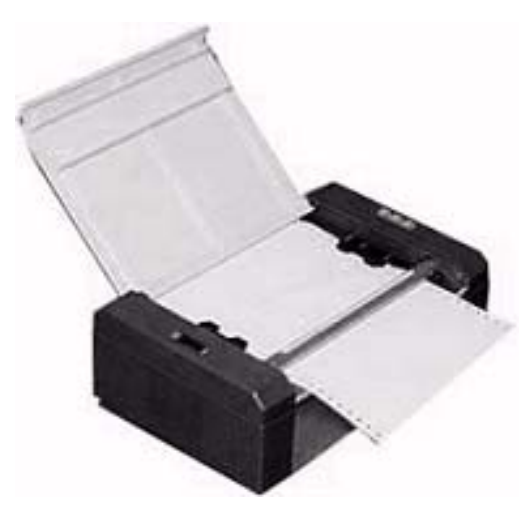

Figura 3 - Impressora Braille

Fonte: http://intervox.nce.ufrj.br/ fabiano/Braille.htm

cilhos e dificuldades. A produÁ,,o de livros em Braille n,o È uma pr·tica das editoras, estando restrita a algumas fundaÁıes ou institutos. O acesso a periÛdicos (jornais e revistas) depende de uma pessoa que esteja disposta a realizar a leitura, o mesmo ocorre com remĖdios, alimentos, entre outros. Dessa forma, somente com o acesso $\ddagger$ inform.tica e, mais especificamente, $\ddagger$ Internet essa dificuldade pode ser minimizada e propiciada a inclus, ,o ao ambiente de informaÁ,,o.

\section{A internet e os PNEEs com limitação visual}

A utilizaÁ,,o da inform tica pelos PNEEs com limitaÁ,o visual e, mais especificamente, da internet È muito recente. O surgimento dos leitores e ampliadores de tela Ė um marco na vida dos usu.rios da inform tica tanto quanto foi o surgimento do Braille.

O evento da sintetizaÁ,,o de voz È um divisor de guas na vida dos profissionais cegos que atuam na inform tica. Afirmo isto porque sou da Època anterior, onde trabalh -vamos sem muitos recursos tÈcnicos. (SOUZA, 2002).

O acesso $\ddagger$ internet pelos PNEEs iniciou lenta e gradativamente, de forma indivi- dual, sem a necessidade de dependíncia de outras pessoas, em virtude de tecnologias assistivas e adaptativas. A dependíncia de outras pessoas para o uso da internet faz com que, muitas vezes, os PNEEs com limitaÁ,,o visual sintam-se inferiorizados no grupo do qual fazem parte, formado por pessoas que $n$ „o possuem essa limitaÁ,,o. As pessoas, com relaÁ,,o aos PNEEs, expressam sentimentos de dualidade: sentem comiseraÁ,,o e procuram auxili--los ou ficam admirados com a desenvoltura que apresentam diante de um computador. Quando o sentimento È de piedade ocorre a superproteÁ,,o, o que dificulta aos PNEEs a superaÁ,o de suas limitaÁıes, impedindo que cresÁam como sujeitos capazes e independentes. $O$ mesmo ocorre quando $\mathrm{h}$ - uma supervaIorizaÁ,,o das tarefas que executam, transformando-os no centro das atenÁıes. A partir do momento que os PNEEs com limitaÁ,,o visual tím a oportunidade de utilizar os mesmos recursos das pessoas que tím vis,, O normal, essas limitaÁıes s,,o bastante diminuldas e praticamente deixam de existir, aumentando a auto-estima e propiciando uma interaÁ,o maior entre os pares, como se pode constatar atravĖs do depoimento de portadores de limitaÁ,o visual: 
A internet mudou drasticamente a minha vida, para melhor, obviamente. De um ìditadorî de textos, passei, eu mesmo, a elabor--los, a corrigi-los, a imprimi-los. Finalmente, consegui ler jornais! Passei a me encontrar em pÈ de igualdade com os normovisuais, quando se trata de usar o correio eletrÙnico e freq, entar as p-ginas web. (A M, 2001).

O computador com leitores de tela e sintetizadores de voz tem sido uma ferramenta indispens vel em meu trabalho e fora dele. Sem este recurso, certamente, eu $n_{\text {, }}$ teria maior autonomia e independíncia ou a mesma desenvoltura no desempenho de minhas atividades. $O$ efeito da inform tica em meu contexto de vida, talvez, possa ser comparado aos efeitos da alfabetizaÁ,o, letramento e escolarizaÁ,,o de uma pessoa que antes, era analfabeta. $\left(S_{i}, 2001\right)$.

Nos dias atuais, existem v-rios leitores e ampliadores de tela, utilizados pelos portadores de limitaÁ,,o visual. Esses programas, segundo Santarosa (2001), contribuem para proporcionar aos PNEEs maior independíncia, qualidade de vida e inclus,,o social.

\section{Os PNEEs com limitação visual e o uso de leitores de tela}

O surgimento dos leitores de tela $\mathrm{E}$ um marco na vida dos PNEEs com limitaÁ,,O visual. Pode-se dizer que È comparado ao surgimento do Braille e a sua inserÁ,,o na cultura letrada. Na dÈcada de 1990, surge no Brasil um programa que possibilitou a utilizaÁ,o da inform tica pelos PNEEs: o Dosvox.

\subsection{Dosvox}

Em 1993, o professor JosÈ Antonio Borges, do Curso de Inform tica da UFRJ, recebeu em sua sala de aula o aluno Marcelo Pimentel, portador de limitaÁ,,o visual.
Em um primeiro momento, Borges pensou em dispens--lo, pois seria muito difìcil ensinar computaÁ „o gr.fica para um aluno com tal limitaÁ,,o. Mas, como a disciplina de ComputaÁ,o Gr.fica È obrigatÛria, o professor propÙs a Marcelo a

criaÁ,,o de um programa sonoro, utilizando um sintetizador de som de baixo custo, que foi montado na prÛpria UFRJ, projeto do Eng. Diogo Takano. Antonio criou as rotinas b-sicas de fala e Marcelo, no decorrer do curso, criou o que hoje Ė o EDIVOX, editor de textos que È utilizado pela comunidade DOSVOX. (BORGES, 2001).

Surge ent,„o o Dosvox, um sistema operacional completo em IÌngua portuguesa que possui um editor (edivox) e leitor de textos, imprime textos em Braille, possui diversos programas (mais de setenta), como: cartavox (correio eletrùnico), papovox (batepapo), webvox (para navegar na web), intervox (criaÁ,,o de homepages), calcuvox (calculadora), cheqvox (para preencher cheques), alĖm de jogos e muito mais. Todos os dias est, ,o sendo realizados ajustes e adequaÁıes. As listas de discuss, ,o Dosvox e Voxtec existem para que as d'vidas em relaÁ,,o ao sistema sejam dirimidas e como espaÁo para os usu·rios opinarem, sugerindo alteraÁıes, que $\vee$ „o sendo implementadas dentro das possibilidades e adequaÁıes do sistema.

Quando o programa È iniciado, apÛs os crÈditos, ouve-se uma voz que diz: Dosvox, o que vocí deseja? A partir desta pergunta, teclando $\mathrm{F} 1$, o usu-rio pode realizar diversas operaÁıes, desde ler e editar um texto, atÈ criar uma p·gina para internet ou construir um texto no MicrosoftWord, utilizando o WordUtil. 


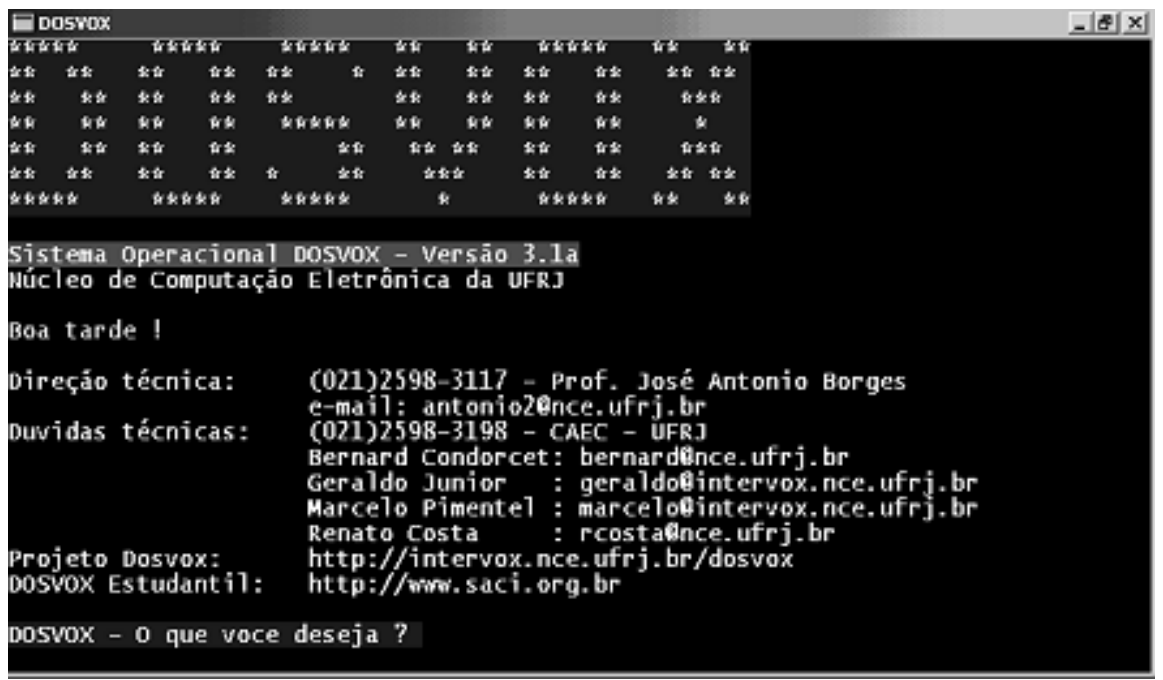

Figura 4 - Tela inicial do Sistema DOSVOX

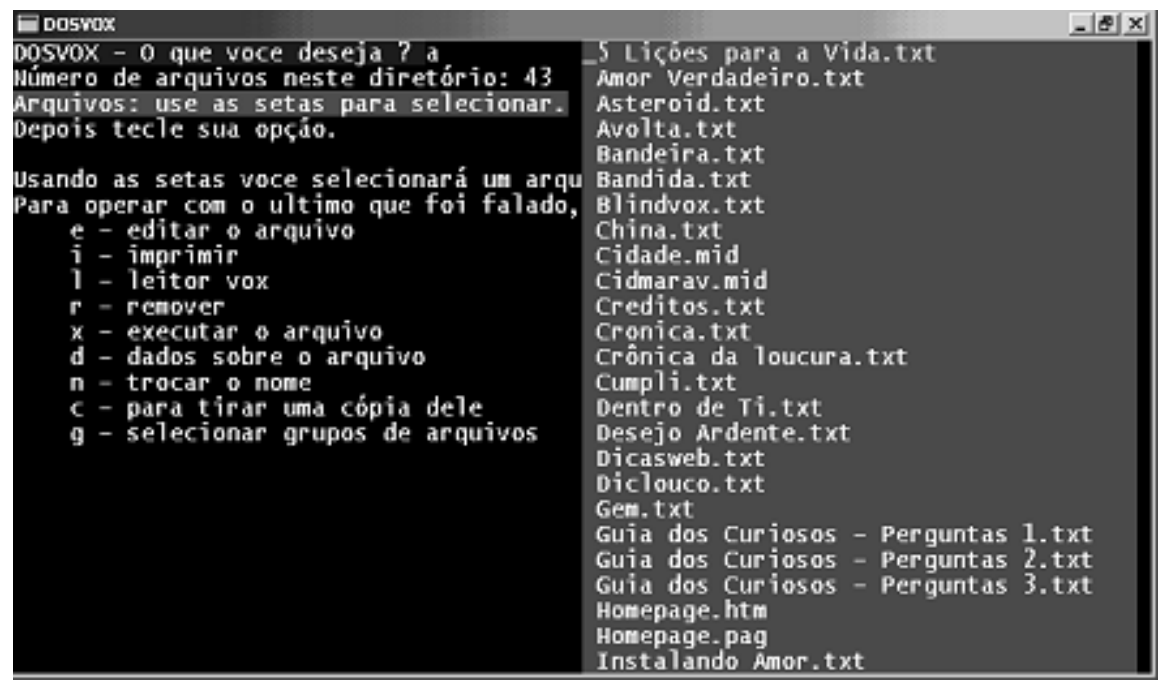

Figura 5 - OpÁıes do Dosvox

Segundo Porto (2001, p.43), dentre as diversas vantagens do uso do Dosvox, sistema que È utilizado por mais de quatro mil usu.rios, no Brasil e demais pal̀ses de Lìngua Portuguesa, podemos citar:

¿fala em portuguís: foi o primeiro desenvolvimento para cegos no mundo cuja sìntese de voz se d· em idioma portuguís (o n' mero de cegos brasileiros que dominam o idioma inglís ainda È mìnimo);

Loferece um alto nlvel de interatividade: seu desenvolvimento est - baseado no que chamamos de interface especializada, havendo a preocupaÁ,,o em reduzir-se ao $\mathrm{m} \cdot x i m o$ 
qualquer comprometimento tÈcnico em nìveis elevados (o Dosvox praticamente ìconversaî com seus usu·rios);

¿custo: atualmente existe uma vers „o simplificada disponl̀vel gratuitamente na www, e mesmo sua vers,o completa ou ìprûî sempre apresentou-se acessìvel sob o ponto de vista financeiro, realista com as condiÁıes de compra ou poder aquisitivo dos deficientes visuais brasileiros (o custo È aproximadamente $15 \%$ menor em relaÁ, o a outros desenvolvimentos do gínero).

O projeto tem um grande impacto social pelo benefìcio que ele traz aos deficientes visuais, abrindo novas perspectivas de trabalho e de comunicaÁ,o. (BORGES, 2001)

\subsection{Virtual Vision}

O Virtual Vision (VV) È um leitor de telas, desenvolvido no Brasil pela Micropower. ... um programa que pode ser utilizado no ambiente Windows e permite navegaÁ,,o na internet pelo Internet Explorer. Apresenta uma Ûtima qualidade de voz e seu idioma È o portuguís.

A primeira vers „, o foi lanÁada em janeiro de 1998, para Windows 95, Office 95 e Internet Explorer 3.02. Em abril de 1998, o Bradesco procura a Micropower para desenvolver o Bradesco Net e, em agosto do mesmo ano, È lanÁado o Bradesco Net Internet Banking para deficientes visuais. Em setembro, a FundaÁ,o Bradesco, a LBV e o Prodam iniciam um curso para invisuais e, sob a coordenaÁ,,o da Micropower e da FundaÁ,,o Bradesco, È formado um grupo de trabalho chamado CapacitaÁ,o e Empregabilidade para os Deficientes Visuais. Em marÁo de 1999, a Faculdade de EducaÁ,,O da USP inicia a capacitaÁ,,o no uso do VV pelos alunos do curso de EducaÁ,,o Especial. Em setembro, a MicroPower lanÁa a vers,,o 2.0 do Virtual Vision, para Windows 95 e 98, Office 95 e 97 e Internet Explorer 5.0.

Na criaÁ,,o do programa surgiu tambÈm a lista de discuss,,o Virtual Vision. No entanto, em 10 de setembro de 1999 , essa lista passou a ter um moderador, Anderson Farias, portador de limitaÁ,o visual. A lista foi criada para orientaÁıes sobre o uso do sistema e para trocas de informaÁıes sobre internet e assuntos relacionados $\ddagger$ limitaÁ,,o visual.

O Virtual Vision È um programa de $\mathrm{f} \cdot \mathrm{cil}$ utilizaÁ,,o. Ao ser ativado, anuncia que est pronto para ser utilizado e ouve-se a seguinte frase: Virtual Vision est- ativado. Como o invisual n,o utiliza mouse e s,o muito utilizados os atalhos do teclado, È possìvel configurar o painel de controle do programa para as diversas possibilidades que oferece (vide Figura 6).

Dentre as vantagens do uso do Virtual Vision, pode-se destacar:

$\Sigma$ dispensa sintetizador externo, pois utiliza apenas a placa de som;

$\Sigma$ È auto-instal-vel;

$\Sigma$ funciona no ambiente Windows e nos aplicativos do Office, permite navegar pela internet no Internet Explorer, sendo possìvel utilizar os programas de e-mail, de OCR, etc.; $\Sigma$ a pron' ncia das palavras pode ser feita letra por letra, palavra por palavra, par.grafo por par.grafo ou o texto todo;

$\Sigma$ possui um mÛdulo de treinamento falado; ¿informa detalhes sobre os controles do Windows, tais como: tipo de controle, estado, etc;

$\Sigma$ permite a leitura de $p \cdot g i n a s$ da internet citando, inclusive, os links para outras $p \cdot g i n a s$. 


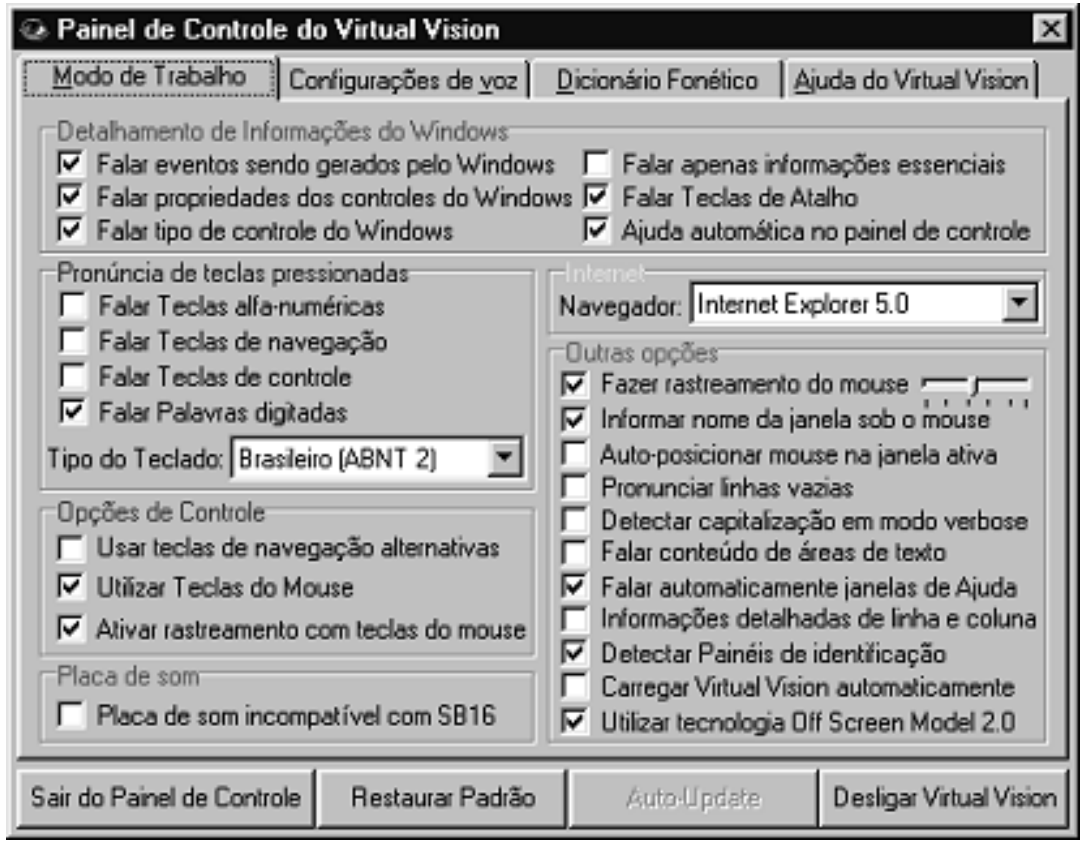

Figura 6 ñ Painel de controle do Virtual Vision (VV)

\section{Os PNEEs com limitação visual e o uso dos ampliadores de tela}

Para as pessoas que possuem baixa vis,,o, existem os ampliadores de tela. Nos editores de texto, por exemplo, È possìvel aumentar a fonte para que a pessoa possa ler o que est · escrito. No entanto, existem programas especlificos que oferecem esse recurso, como o programa LentePro, que, segundo Borges (2000), foi criado pelo projeto Dosvox, para ajudar o usu·rio Windows com vis,,o subnormal que $\mathrm{n}, 0$ tem recursos suficientes para comprar um programa mais sofisticado e caro no exterior. AtravÈs desse programa, o que aparece na tela Ė ampliado numa janela, o que permite a percepÁ,,o de todos os detalhes, mesmo por aqueles deficientes com grau muito baixo de acuidade visual. O programa È de simples utilizaÁ,,o e de tamanho pequeno, o que permite a gravaÁ,o em um ' nico disquete. Isto permite que um deficiente visual possa carregar consigo o programa e utiliz--lo em qualquer computador.

O LentePro tem um conjunto grande de alternativas de configuraÁ,,o. Ele pode ser configurado, opcionalmente, atravĖs do teclado, o que diminui a necessidade de configuraÁ,o visual.

AlÈm desse programa existem outros ampliadores de tela como:

ZLunar 95 ó Aumenta os textos e gr·ficos no Windows 95 em atÈ dezesseis vezes e trabalha com dezesseis cores. Possui a facilidade da divis, ,o em partes da tela, o que permite o aumento de porÁıes distintas da tela. Segue automaticamente e magnifica 
menus, highlights, cursores, etc. Apresenta um modo inteligente de suavizaÁ,,o de textos, que melhora a legibilidade de fontes dos sistemas ampliados.

$\Sigma$ ZoomText Windows ó Amplia de duas a vinte e seis vezes, com escolha de trís fontes com cantos suavizados. Magnifica a tela dos processadores de texto, planilhas, programas de bancos de dados, etc. Apresenta estilos de fontes, seguimento de cursor, rolagem de tela, visualizaÁ,,o e revis,,o em qualquer direÁ,,o, zoom por linha, janela ou tela inteira. Suporta SVGA atÈ 1024 x 768 e 256 cores. O usu rio pode criar suas prÛprias fontes escalon veis. ... possìvel trocar cores e outros controles avanÁados.

¿Magic Deluxe ó Apresenta encaixotamento e empilhamento. Segue suavemente o cursor, o mouse e as atividades da tela. Inclui um cursor grande e localizadores de tela embutidos. Proví suporte de marcaÁıes, suporte $\ddagger$ tela dividida, oito estilos de fonte $e$ amplia em atÈ doze vezes. A vers,,o Lite, mais barata, magnifica textos e janelas duas vezes.

¿LP DOS Deluxe ó Totalmente interativo. Magnifica os textos atĖ dezesseis vezes. ... controlado por menus e teclas de funÁ, ,O. Segue o cursor automaticamente. Pode trocar as cores do fundo.

$\Sigma$ Visability ó Software de simulaÁ,,o de CCTV. O material È lido no scanner e a informaÁ,o mostrada ampliada na tela, como no CCTV, de forma ainda mais vers til. Permite a ampliaÁ,,o de jornais, cartas manuscritas e figuras no computador. Possui nìveis de magnificaÁ,,o de duas a trinta e duas vezes (oito nìveis). Possibilidade de impress„,O do material ampliado.

\section{$6 \quad 0$ ambiente de aprendizagem mediado por computador}

A utilizaÁ,o dessas tecnologias possibilita o uso da inform tica e da internet com fins pedag Ûgicos, bem como a inclus,,o dos PNEEs em ambientes interativos. O Ambiente de Aprendizagem Mediado por Computador (AAMC) envolve v-rios elementos para o processo de ensinar e de aprender: o educador, os aprendizes, a colaboraÁ,,o, a cooperaÁ,o e as ferramentas.

No AAMC o educador tem o papel de mediador, propiciando o exercl̀cio da colaboraÁ,,o e da cooperaÁ,,o dos envolvidos nas atividades realizadas. Nesse ambiente, os alunos tornam-se o centro do processo, sendo respons -veis pela sua aprendizagem e co-respons veis pela aprendizagem do grupo do qual fazem parte.

[...] a atividade de aprendizagem pode ser oportunizada de forma cooperativa se os alunos estiverem estritamente ligados de maneira que cada um deles saiba e sinta que o Íxito pessoal ajuda os colegas aos quais est- unido para alcanÁar o seu, os resultados almejados por cada membro do grupo s,,o, portanto, benĖficos para os outros membros com os quais est interagindo cooperativamente. (CASTENEDA; FIGUEROA, 1994, p. 43).

Alguns autores fazem uso dos termos ìcooperaÁ,,Oî e ìcolaboraÁ,,oî com significados indistintos. Outros salientam a diferenÁa entre um termo e outro. Alguns definem, outros conceituam. No entanto, na AAMC a colaboraÁ,,o e a cooperaÁ,,o tím funÁıes distintas.

[...] colaborar est $\cdot$ relacionado $\ddagger$ contribuiÁ,,O enquanto cooperar envolve $\mathrm{v}$-rios processos ñ comunicaÁ,,o, negociaÁ,,O, corealizaÁ,o e compartilhamento [...] co-realizaÁ,o È um trabalho cooperativo em essíncia ñ È o fazer junto, em conjunto. ... 
o co-projetar, co-desenvolver, co-realizar e co-avaliar. O prefixo ìcoî implica em uma sÈrie de requisitos para que ocorra uma atividade em conjunto. (BARROS, 1994, p.27-28).

As ferramentas utilizadas no AAMC devem ser selecionadas pelo educador, tendo em vista o perfil, as caracterl̀sticas e as necessidades do grupo, para o bom desempenho das atividades sìncronas e assìncronas desenvolvidas, bem como a preocupaÁ,o para que as mesmas propiciem um ambiente de interaÁ,,o e de acesso ‡ informaÁ,o.

A interaÁ,,o entre portadores de limitaÁ,,o visual e pessoas com vis,,o normal deve ser um processo de di-logo, de colaboraÁ,o. Existe a necessidade de superaÁ,,o de dificuldades, como a leitura em Braille por pessoas que $\mathrm{n}_{\text {, }}$ o dominam o sistema, o acesso ao computador por parte dos portadores de limitaÁ,,o visual, a mudanÁa de papÈis, quando o professor deixa de ser um transmissor de conhecimentos e o aluno o receptor, dentre outros.

Para que ocorra a aprendizagem, È necess rio um processo de troca com o outro. A aprendizagem colaborativa apoiada por computador deve ser uma estratĖgia educativa na qual dois ou mais sujeitos constroem o seu conhecimento a partir da discus$\mathrm{s}$, O, do di·logo, da reflex,o, da tomada de decis,,o, tendo como mediador o computador. Para que esse processo ocorra, professores e alunos devem estar dispostos a colaborar e construir conjuntamente.

... necess rio que o portador de limitaÁ,o visual supere as dificuldades e passe a ter uma maior autonomia. No entanto, isto sÛ ser. possìvel com a colaboraÁ,,o do outro. Ser a oportunidade de ambos se co- nhecerem melhor, descobrirem uns nos outros as suas habilidades e a contribuiÁ,o que cada um pode oferecer ao grupo em um processo de aprendizagem e construÁ,,o de conhecimento.

Para que o grupo se fortaleÁa e consiga trabalhar de forma cooperativa e colaborativa È necess-rio o di-logo, a troca, a construÁ,,o conjunta. Os integrantes do grupo devem ter objetivos comuns, todos devem contribuir unscomos outros. Cadaintegrantedeve ser respons.vel pelo seu aprendizado e pelo aprendizado do outro. O grupo deve sempre retomar as atividades, fazendo uma avaliaÁ,,O do processo, verificando pontos que devem ser aprimorados e propiciando uma reflex,,O diante do processo de construÁ,,o colaborativa. No entanto, o processo ocorrer- somente se forem utilizadas as ferramentas que possibilitem essa colaboraÁ,o.

O Ambiente de Aprendizagem Mediado por Computador deve permitir a cooperaÁ,,o, atravĖs da interaÁ,,o dos alunos com o ambiente, com o educador, com os pares e com as pessoas envolvidas no processo de ensino e de aprendizagem, possibilitando a construÁ,„o do conhecimento.

[...] o ambiente deve ser voltado para aprendizagem e permitir que o aluno torne-se um agente ativo que (re) constrûi seu conhecimento na interaÁ,o sujeitoobjeto. Nesse pressuposto, o computador (e o ambiente telem tico em particular) torna-se uma ferramenta que oportuniza a interaÁ,,o entre o sujeito-objeto e entre sujeitos-sujeitos. Assim sua estrutura centra-se no aluno e nas suas necessidades e crenÁas, sendo sistemas abertos nos quais o conte do n,,o È prè-determinado. (PASSERINO; SANTAROSA, 2003).

No caso dos portadores de limitaÁ,,O visual, deve haver uma preocupaÁ,o em re- 
laÁ,, $¥$ acessibilidade. A maioria dos sites pode ser acessìvel, principalmente se construldos em html. Deve haver um cuidado especial com a utilizaÁ,,o de Flash e Applets Java, que tornam os sites inacessìveis, a utilizaÁ,,o de frames e tabelas, que dificultam a navegaÁ,,o; as figuras que devem incluir o ìalternate nameî, o atributo ìaltî em html, com a descriÁ,,O, para que os PNEEs com limitaÁ,o visual saibam do que se trata e, quando a figura for um link, ser acompanhada da funÁ,o que simboliza. O mesmo cuidado em relaÁ,,O com as ferramentas de interaÁ,,o, como o chat que deve ser adaptado. Um exemplo Ė o papovox, que Ė um programa de bate-papo falado para portadores de limitaÁ,,o visual e pessoas com vis,,o normal.

A construÁ,o de um ambiente cooperativo de aprendizagem computacional deve estar relacionada $\ddagger$ s necessidades dos alunos. Como uma das grandes dificuldades È o acesso a materiais como livros did-ticos e periÛdicos, a utilizaÁ,,o da internet passa a ser uma fonte de informaÁ,o e pesquisa.

O ambiente dever- possuir ferramentas de comunicaÁ,,o mediadas por computador como listas de discuss, ,, , bate-papo falado, p.ginas interativas, dentre outras. Deve haver a possibilidade de comunicaÁ,,o com especialistas nas ' reas pesquisadas, propiciando a troca com pessoas mais experientes. Para Vygotsky (1984), apud Rego (1995, p.110), ìconstruir conhecimentos implica numa aÁ,,o partilhada, j· que È atravÈs dos outros que as relaÁıs entre sujeito e objeto s, o estabelecidasî.

Segundo Franco (2003) ì [...] a construÁ,,o do conhecimento sÛ se d· nas trocas com os outros, e o saber diferenciado do professor torna-se elemento essencial nes- ta construÁ,,O. Portanto, n,o deixemos de lado a necess ria dialogicidade da relaÁ,,o professor-alunosî.

Dessa forma, professores e alunos poder,,O construir juntos e buscar a soluÁ,,O de problemas, a superaÁ,o de dificuldades, em um processo de construÁ,o e (re)construÁ,,o de conhecimento, propiciando uma oportunidade de inclus,,O dos PNEEs portadores de limitaÁ,o visual.

\section{Considerações finais}

Vive-se em uma sociedade marcada pela diversidade e pela exclus,,o social. Por isso, a inclus,,o digital deve ser uma realidade em todos os segmentos dessa sociedade. Os portadores de limitaÁ,o visual, com o surgimento do Braille, passaram a fazer parte da cultura letrada, mas com uma escrita prÛpria, que pode ser utilizada somente por quem a domina. Com a inform·tica, eles passaram a fazer uso das tecnologias computacionais, adaptadas para atender as suas necessidades, atravÈs da utilizaÁ,o dos mesmos recursos que as pessoas com vis,,o normal.

Apesar da existíncia dessas tecnologias, poucas pessoas tím conhecimento e acesso $\ddagger$ s ferramentas adaptativas e assistivas. Por outro lado, a escola, atravÈs de seus professores, deve estar preparada para receber e integrar os alunos PNEEs em todo o processo de ensino e de aprendizagem. Esses alunos $\mathrm{n}, 0$ representam a ìt·bula rasaî, mas tím muito a contribuir, a colaborar, a cooperar e a compartiIhar em todas as atividades vivenciadas dentro e fora da sala de aula.

As escolas que possuem Ambientes de Aprendizagem Mediados por Computa- 
dor, em muitos casos, acabam excluindo os PNEEs das atividades realizadas nos laboratÛrios de inform tica, pois os professores $\mathrm{n}$, ,o est,,o preparados nem estimulados para orient--los no uso adequado das ferramentas assistivas e adaptativas, inclusive por que desconhecem a sua existíncia. O mesmo ocorre em relaÁ,,o łs pessoas respons.veis pela criaÁ,o das p·ginas, programas, dentre outros, que n,o tím uma preocupaÁ,,o em relaÁ,o $\neq$ acessibilidade e $\ddagger$ interaÁ,,o. Existem normas e padrıes que poderiam ser seguidos para tornar essas ferramentas acessìveis a todos, pois, muitas vezes, ao navegar pela internet utilizando leitores de telas, tornam-se totalmente inacessìveis, impossibilitando o acesso ‡ informaÁ,,o.

O acesso łs fontes de informaÁ,,o e a Ambientes de Aprendizagem Mediados por Computador ser - a porta de entrada para a inclus,,o social. Se forem disponibilizadas aos PNEEs as ferramentas que propiciam a colaboraÁ,,O e a cooperaÁ,o ocorrer a interaÁ,o entre os pares e, destes com os professores, resultando a construÁ,o do conhecimento. O uso dessas ferramentas permitir. que os PNEEs tenham as mesmas oportunidades que os demais usu.rios da inform tica, sendo considerados como iguais em uma sociedade de tantas diferenÁas.

\section{ReferÍncias}

A.M. Internet. [mensagem pessoal]. Mensagem recebida por<estabel@cpovo.net>em 30 nov. 2001.

BARROS, L. Suporte a Ambientes Distribuídos para Aprendizagem Cooperativa. 1994.120f. Tese (Doutorado em Inform·tica) ñ Universidade Federal do Rio de Janeiro, Rio de Janeiro, 1994.

BELARMINO, J. As Bibliotecas Universitárias e o Acesso à Informação pelos Indivíduos Cegos. Disponlvel em: <http://intervox.nce.ufrj.br/ joana/textos/tecni12.html>. Acesso em: 2 abr. 2003.

Braille. [mensagem pessoal]. Mensagem recebida por<estabel@cpovo.net>em 3 nov. 2001.

As Novas Tecnologias e a Desbrailização:mito ou realidade? Disponlvel em: <http://intervox.nce.ufrj.br/ joana/textos/tecnol08.html> Acesso em: 14 nov. 2001.

BENGALA BRANCA. Produtos. Disponlvel em: <http://www.bengalabranca.com.br>Acesso em: 20 nov. 2001.

BORGES, J. A. Ampliadores de Tela de Computador:†uma vis,„o geral. Disponìvel em: <http://www.baixavisao.com.br/artigos_bvisao/ampliadores_tela.htm >.Acesso em 20 nov. 2001.

Histórico do Dosvox. Disponlvel em:<http://caec.nce.ufrj.br/dosvox/histdvox.html>Acesso em 17 nov. 2001.

BRASIL. MinistÈrio da EducaÁ,o, Secretaria de EducaÁ,o a Dist,ncia. Educação Especial: tendências atuais. Brasilia: SEED, 1999.

CASTANEDA, M.; FIGUEROA, M. Contexto de Ensenanza: InteraciÛn y Cooperatividad en el Aprendizaje. In: Tecnologia e Comunicación Educativa, Caribe, p.59-65, jun. 94.

DECLARA «VO MUNDIAL SOBRE EDUCA «VO PARA TODOS. Disponlvel em: <http://www.interlegis.gov.br/processo_legislativo/copy_of_20020319150524/20030620161930/ 20030623105532/view> Acesso em: 20 ago. 2003.

DEMO, P. Conhecimento e Desenvolvimento. In: Educação em Revista, Brasilia, DF, p.12-18, fev.2001. 
ESTABEL, L. B. A Internet Modificando a Vida dos Portadores de Limitação Visual através do uso de Listas de Discussão. 2002. 60f. Trabalho de Conclus,,o de Curso (EspecializaÁ,,o) ñ Faculdade de EducaÁ,,o, Universidade Federal do Rio Grande do Sul, Porto Alegre, 2002.

FRANCO, S. R. K. Educação Através da Pesquisa. Disponl̀vel em: <http://www.nuted.edu.ufrgs.br/ franco/educacaopesquisa.htm> Acesso em: 28 maio 2003.

J.P. Apresentação in Virtual Vision Lista de Discussão. Lista de Discuss,,o do Programa Virtual Vision. Disponlvel em:<virtualvision@yahoogrupos.com.br> Acesso em: 5 set. 2001.

LIMA, F. J. de. Questão de Postura ou de Taxonomia? Uma proposta. S,„o Paulo: USP, 2001 in Dosvox Lista de Discuss,,o.Lista de Discuss,,o do Programa Dosvox da UFRJ. Disponlivel em: <dosvox-l@listas.nce.ufrj.br> Acesso em:5 nov. 2001.

PASSERINO, L. M.; SANTAROSA, L. M. C. Vygotsky e os Ambientes Telemáticos. Disponìvel em: <http://libra.niee.ufrgs.br/ proinesp/amb_dig/index.html>Acesso em:2 abr. 2003.

REGO, T. C. Vygotsky: uma perspectiva histÛrico cultural na educaÁ„o. PetrÛpolis: Vozes, 1995.

ROCHA, H. etal. Ensaio sobre a Problemática da Cegueira: prevenÁ,oñ recuperaÁ,o, reabilitaÁ,o. Belo Horizonte: FundaÁ,,o Hilton Rocha, 1987.

$\mathrm{S}_{\mathfrak{i}}, \mathrm{E} . \mathrm{D} . \mathrm{de}$. Lista Dosvox [mensagem pessoal]. Mensagem recebida por<estabel@cpovo.net> em 3 nov. 2001

SANTAROSA, L. M. C. Ambientes de Aprendizagem Virtuais/Digitais para o Desenvolvimento e a Inclusão de Pessoas com Necessidades Educativas Especiais. Disponlvelem: <http://libra.niee.ufrgs.br/ proinesp/amb_dig/index.html>. Acesso em: 20 fev. 2003.

SILVA, L. A. M. C. Lista VV [mensagem pessoal]. Mensagem recebida por<estabel@cpovo.net>em 14 de nov. 2001.

SOUZA, W. Lista VV [mensagem pessoal]. Mensagem recebida por<estabel@cpovo.neŁem 3 de nov. 2001 VYGOSTKY, L. S. A Formação Social da Mente. S,,o Paulo: Martins Fontes, 1984.

Aceito para publicaÁ,,o em 2003/1

\section{Lizandra Brasil Estabel}

Professora do Curso de Biblioteconomia da FABICO/UFRGS, doutoranda em Inform·tica na EducaÁ,o (PGIE/UFRGS), bibliotec ria do ColĖgio $M$,e de Deus e do Instituto Santa Luzia.

E-mail: estabel@cpovo.net)

Eliane L. da Silva Moro

Professora do Curso de Biblioteconomia da FABICO/UFRGS, especialista em Inform·tica na EducaÁ„,o (PGIE/UFRGS), bibliotec ria.

E-mail:mmoro@adufrgs.ufrgs.br

\section{Lucila Maria Costi Santarosa}

Professora doutora no Curso de PÛs-GraduaÁ,,o em Inform·tica na EducaÁ,,O (PGIE) e do Programa de PÛs-GraduaÁ,,o em EducaÁ,,o (PPGEDU) da UFRGS, pesquisadora IA do CNPq e consultora da SEESP/MEC, presidente da Redespecial-Brasil e coordenadora nacional da RIBIE.

E-mail: lucila.santarosa@ufrgs.br 